\title{
Positive Development in Children and the Precursors of Healthy Life-Styles: The Role of Eating Regularity and Level of Leisure Activity
}

\author{
Silvia Ciairano ${ }^{1}$, Giulia Bardaglio ${ }^{2}$, Emanuela Rabaglietti ${ }^{1}$, Maria Fernanda Vacirca ${ }^{1}$ \\ ${ }^{1}$ Department of Psychology, University of Turin, Turin, Italy; ${ }^{2}$ SUISM - Scuola Universitaria Interfacoltà di Scienze Motorie, Centro \\ Ricerche in Scienze Motorie e Sportive, University of Turin, Turin, Italy. \\ Email: silvia.ciairano@unito.it
}

Received May $15^{\text {th }}, 2010$; revised June $17^{\text {th }}, 2010$; accepted June $21^{\text {st }}, 2010$.

\begin{abstract}
The two sides of children positive development, that is physical and psychological health, have been most often investigated separately. We explored the relationships between not being overweight, respecting relational rules, regularity of eating behavior (eating breakfast) and involvement in active (e.g. playing in team sport) or sedentary (e.g. playing at videogames) leisure activities shared with friends. The study was conducted among 272 Italian children (52\% female; $M$ age $=6.85 \mathrm{yrs})$ using a multi-informant design (children, parents and teachers of physical activity). Hierarchical regression analyses revealed that regularity of eating breakfast (informed by parents) was associated to lower levels of Body Mass Index (BMI) values (objectively measured) in girls. Involvement in sedentary leisure activity with friends (informed by children) was negatively associated with respecting relational rules (evaluated by teachers of physical activity) in boys. Thus, more or less active leisure activity and more or less healthy eating behaviour have some potential relevance for present and future general adjustment of boys and girls, and not only for their physical condition. Implications for educational interventions are discussed.
\end{abstract}

Keywords: Children, Life-Style, Overweight, Leisure Activities

\section{Introduction}

There are at least two distinct sides to positive development [1-3]. The first side of positive development refers to physical health. In absence of other relevant pathologies, we know that an individual's body weight is a good indicator of their present and future physical health condition. We know that the problem of childhood obesity is rapidly increasing in all Western societies [4]. The manifestation of childhood obesity is a very strong risk factor for subsequent involvement in Type II Diabetes and for various other pathologies, such as cardio-vascular diseases [5].

The second side of positive development refers to psychological health. Plenty of research has shown that a good indicator of present and future psychological health is an individual's capacity to respect social rules, especially those related to relational aspects during childhood. For instance, breaking the basic relational rule of not being aggressive towards peers has been seen to result in future maladjustment.
Nowadays adults often complain about having difficulty in getting their children to respect social rules. The lack of respect of social rules may assume various configurations, from disobedience to aggressiveness and victimization of peers. We note that public opinion and the media are currently paying great attention to the latter given the great negative, physical and psychological consequences for the victims.

Although the two phenomena of obesity and transgression of rules are usually investigated separately, we would like to investigate them in the same study. We know that there have been very few previous attempts to investigate both phenomena concurrently. To our knowledge one of the few studies that have analyzed both variables in a single study is the very recent research by Giletta et al. [6] that found a relationship between being overweight and bullying peers in a sample of Dutch adolescents.

We know that the phenomena of obesity and of rules transgression usually increases sharply after puberty and 
during early adolescence. However we are more interested in investigating these phenomena during childhood, when they are probably at their inception.

In this study we are curious to investigate whether or not there are similarities between the predictors of the two phenomena. Specifically, we think that in both cases a crucial role is fulfilled by sedentary activity. We have good theoretical reasons for anticipating the risk role of sedentary activity, although the theories we would like to employ have seldom been used before to explain behaviour in children. At first we will refer to the problem behaviour theory of Jessor et al. 's [7,8]. Secondly we will refer to the constructs of organized activity and of initiative of Larson [9].

\subsection{The Precursors of Healthy Life Styles in Childhood according to the Problem Behavior Theory by Jessor}

Although the theory by Jessor et al. is very well-known in the field of adolescent psychology, we will summarize it very briefly in order to individuate the aspects that can be adapted to earlier developmental phases, and especially to individuate some precursors of more or less healthy life-styles in childhood. Jessor and colleagues [7] theoretical framework consists of three main notions. The first notion, which is conceptually similar to the idea of development as action in context [10], concerns the fact that risk behavior, and especially that by adolescents, is not a pathological answer to internal drives or external stimulus. Rather, risk behavior depends upon the complex balance across time among the system of the person, which includes self-perception, attitudes, expectations, and values; the system of the perceived environment, which considers the relationships of the individual with the three main developmental contexts of family, peers, and school; and the system of behavior, which considers both risk behavior and conventional behavior. Also in the case of the children we can reasonably suppose that behavior depends upon the complex balance across time among physical and psychological individual characteristics, relationships with the developmental contexts of family, peers, and school and behavior themselves, for instance more active and/or more sedentary activity.

The second notion has to do with the fact that in each one of the personality, perceived environment or behavior systems some aspects are expected to fulfill the role of protective factors, while other factors are expected to work as risk factors. For instance respecting relational rules and good quality of the relationships with the peers, are expected to fulfill the role of protective factors, while other factors, as sedentary activity, are expected to work as risk factors. Generally speaking, risk factors may weaken individuals resolve not to engage in different kinds of risk behaviors. The three protective factors in the model by Jessor et al. [11] are models protection, controls protection, and support protection. Models protection encompasses parents' and friends' models for health behavior. Health behaviors include diet and exercise, which can be relevant for children too. Conventional behaviors also include participation in sports. Controls protection also includes the parental controls over children behavior, such s promoting regularity of eating and the respect of relational rules during childhood. Support protection includes support from family, friends, and other important adults in the children' lives. Jessor et al.'s $[7,8]$ theoretical model emphasizes that the balance among risk and protective factors contributes to the likelihood that the individual will engage in more health or risky behavior, thus to his/her adjustment. The third notion of the theoretical model by Jessor et al. [7] concerns the presence of a constellation or a syndrome of risk behavior, that is to say that risk behaviors are likely to co-occur: an individual who is engaged in one risk behavior (e.g., disturbed or irregular eating), also is likely to be engaged in other risk behaviors (e.g., transgression).

\subsection{Leisure and the Development of Initiative}

We also refer to some theoretical cues drawn from the literature about the potential role of organized leisure activity, and among them especially sport, in developing the individual capacity of initiative and in contributing to the positive development of people.

Larson [12] has defined the time of leisure as the fourth developmental context, together with the family, the school and the peer group, for children and adolescents. Leisure activity can be distinguished in organized or structured and in not organized and un-structured. The structured activities, which are generally leaded by adults, are carefully organized for realizing specific tasks and for acquiring capabilities and competences (such as in sport). The not organized and un-structured activity are all those kinds of situations that do not pursue the realization of specific tasks, the supervision of adults may lack and they happen outside any institutional context of meeting [13]. However both kinds of activity can be more active (for instance playing at team sport) or more sedentary (for instance playing at videogames). All children and adolescents are more involved at different extent in these four kinds of activities. Participation at organized and active activity has been generally found associated with a more positive development of the individuals. In particular, various studies underlined that the adolescents who participate at organized activity are less involved in risk behavior and have higher levels of self-efficacy $[14,15]$.

Among all these activities, we are particularly interested in the kind of activity that can promote in children and adolescents the personal capacity of initiative. Ac- 
cording to Larson [9] the capacity of initiative is essential for developing as full members of our complex society and it will become even more important in the near future. However, despite its importance, children and adolescents have very few chances of learning and exercising this capability. In fact the normal experience of children and adolescents at school and also during the leisure time usually does not include all the necessary conditions for learning it. In our opinion, this is particularly true when they are involved in sedentary leisure activity. The context that is more adequate for developing the capability of initiative is that of volunteer but structured activity, such as that of sport, in which children and adolescents can experiment the rare combination between intrinsic motivation and concentration.

In agreement with Larson and also with other scholars [16] we believe that leisure activity, and particularly sport activity, that are organized accordingly with the above mentioned principles could represent a context particularly favorable at the positive development of children and youths. However, we also think that the combination of positive psychological conditions, intrinsic motivation and concentration could be individuated, at least partially, in more spontaneous kind of leisure activity. For instance when children meet in the courts of their houses during their free time and they organize spontaneously and by themselves for playing team sport with their peers. On the contrary we think that especially during childhood participation at un-structured and sedentary kinds of leisure activity could have negative consequences not only for the future development of the capability of initiative but also for the present and future physical health, that is in term of overweight, and for the psychosocial adjustment, especially in term of diminished possibility of developing social competence including that of respecting relational rules. A sedentary and passive type of leisure activity is unlikely to represent the adequate context for promoting social competence [17].

\subsection{The Present Study}

On the basis of the above mentioned theories, we expected that regularity of eating, and spontaneous leisure activity, active or sedentary, would emerge as possible precursors of more or less healthy life-styles during childhood. More specifically, we expected to find that regularity of eating (in terms of breakfast) and active spontaneous leisure activity would work as protective factors and thus would be associated to a more positive development of children in terms of both being not overweight and being able to respect relational rules in a social context, which we considered as two possible indicators of adjustment. Conversely, we expected to find that sedentary spontaneous leisure activity would work as a risk factor and that it would be associated with a less positive child development, in terms of being overweight and not respecting relational rules, which we considered lack of children adjustment.

However, considering that we were unaware of previous research about similar relationships between potential precursors of healthy life-style and positive development or adjustment in childhood, our study was mainly exploratory, especially with respect to the possibility of observing gender differences. In summary we asked two key questions in this study:

1) Were there mean level differences between boys and girls on measures of physical and psychosocial positive development, and precursors of protective factors and risk factors? As anticipated this is mainly an exploratory study, however we would expect to find few differences. More specifically, and on the bases of studies on adolescents, we anticipated that girls would be more likely to respect relational rules [18] and that boys would be more likely to be involved in both sedentary and active kinds of leisure activity [19,20].

2) Did the same precursors of risk and protective factors account for variation in indicators of positive development in children in both the subsamples of boys and girls? We expected to find similar relationships with respect to both indicators of childhood adjustment in boys and girls. However, considering that the emergence of eating problems at later stages of development are generally more common in girls and conversely that transgression is more diffuse among boys, we expected to find some gender differences with respect to the patterns of relationships between indicators of adjustment and precursors of protective and risk factors.

\section{Method}

\subsection{Participants}

Participants were 272 children aged 6 to $8(M=6.85, S D$ $=0.71 ; 53 \%$ females $)$ attending six primary schools in Cuneo, Italy. The majority of the children's parents $(43 \%$ of fathers and $57 \%$ of mothers) had a high school diploma. $24 \%$ of fathers and $17 \%$ of mothers had a university degree. Most parents (99\% of fathers, $78 \%$ of mothers) were employed. Regarding family structure, $92 \%$ of the parents lived together, and $8 \%$ were separated or divorced. These figures of socio-demographic information are similar to those found in the general population from the same Italian province, which is one among those at lower level of un-employment in Italy [21].

\subsection{Procedure}

As previously stated, the study was conducted in six primary schools in Cuneo, Italy. A random sample of public primary schools was invited to participate in the study and all schools contacted agreed to do so. Classes 
within the schools were then randomly selected and all classes contacted accepted to take part in the study. Finally, all the parents contacted provided consent for children to participate, and children themselves assented to participate in accordance with Italian law and the ethical code of the Professional Psychologists Association in Italy. In addition, the children took the questionnaire home to their parents. $96 \%$ of the parents completed the questionnaire about socio-demographic information and children's behavior and returned it back the following week; the questionnaire was completed $57 \%$ by mothers, $13 \%$ by fathers and $26 \%$ by both parents. We did not find any relevant differences between the children of the parents who filled in the questionnaire and those who did not with respect to the other information under study.

Finally, the teachers of physical activity, who were 6 and who were all graduated in physical activity and specially trained for coaching children, observed and registered the children's behavior about respecting relational rules as regard to teachers, and classmates. This was accomplished by way of a check-list and they measured the children's Body Mass Index by way of an electronic balance during the initial session of an experimental study which will ultimately evaluate the effectiveness of the introduction of a special program of physical activity which we will consider in a future paper.

Both the children's and parents' questionnaires took approximately 30 minutes to complete. The observation of children' behavior took approximately 60 minutes to complete. Both children and parents were assured of confidentiality and anonymity. Teachers were not present in the classroom during the questionnaire administration for the children. No incentives were offered for participation; however $100 \%$ of the children completed the questionnaires.

\subsection{Measures}

Leisure activity. Sedentary and active leisure activity were assessed using two simplified items (selected because of children's young age) that asked children to answer whether or not they usually spend time with their friends, 1) doing active things such as playing in the court or at team sport; 2) doing sedentary things such as playing at videogames or watching $\mathrm{TV}$.

Regularity of breakfast. The regularity of children' breakfast was assessed using one item that asked parents whether their children usually eat breakfast and ranging from 1 - Never to 4 - Always.

Respect of relational rules by children. This item was assessed on the basis of the evaluation of the teachers of physical activity using a simple check-list. The check-list contains the names of the children on the rows and three columns with the space for the information about respect of relational rules. During one hour of physical activity and working in couples, scored each child was with 3 if he/she always respected some relational rules, with 2 if he/she respected them only partially and with 1 if he/she never respected them. The relational rules considered in the check-list were about: a) respecting the turn of the others; b) giving the other children enough space to move for doing their exercises. The score attributed by each teacher to each child were summed up.

Body Mass Index. This item was assessed by the teachers of physical activity by way of an electronic balance, which measured height and weight and calculated the Body Mass Index automatically.

\section{Results}

\subsection{Descriptive Analyses}

Table 1 presents descriptive information of the sample and correlations among the variables by gender. To assess for gender differences on the study variables, we used t-tests for independent samples. There were no gender differences in mean levels of Body Mass Index, regularity of breakfast, and active leisure activity. However, girls $(\mathrm{M}=$ $5.75, S D=0.61)$ reported more respect of relational rules than boys $(M=4.86, S D=1.04), t(262)=8.56, p<0.001$. Boys, relative to girls, reported higher levels of sedentary leisure activity $(M=0.76, S D=0.43$ for boys; $M=0.66$, $S D=0.48$, for girls; $t(270)=1.97, \mathrm{p}<0.05)$.

Furthermore girls and boys showed a different pattern of inter-correlations among indicators of adjustment and precursors of healthy life-style. In girls Body Mass Index was negatively associated with regularity of breakfast and positively associated with sedentary leisure activity. In boys Body Mass Index and respect of relational rules were negatively associated between each other, and respect of relational rules was negatively associated with sedentary leisure activity. Also on the basis of these different patterns of relationships, we decided to perform the following analyses separately for boys and girls.

\subsection{Relationships among Body Mass Index, Respect of Relational Rules, Eating Behavior and Leisure Activity}

To test the relationships among Body Mass Index and respect of relational rules, and among regularity of breakfast, and active and sedentary leisure activity, we used Hierarchical Linear Regression Models.

In the regression models (one for each outcome and separately for boys and girls), we entered regularity of breakfast in Step 1, active leisure activity in Step 2, and finally sedentary leisure activity in Step 3.

\subsection{Children' Body Mass Index and Precursors of Life-Style}

Only the model with girls achieved a significant propor- 
tion of explained variance: $\mathrm{R}^{2}=0.11, \mathrm{~F}(3,140)=5.67, p$ $<0.0001$ with the coefficient of regularity of breakfast significant (see Table 2). This means that in the female sample, children who were more regular in doing their breakfast also had lower Body Mass Index that is they were less likely to be overweight.

In the male sample, we found a positive relationship between Body Mass Index and sedentary leisure activity. However, this relationship did not reach significance.

\subsection{Children's Respect of Relational Rules and Precursors of Life-Style}

Only the model with boys achieved a significant proportion of explained variance: $\mathrm{R}^{2}=0.08, \mathrm{~F}(3,116)=3.35, p$ $<0.02$ with the coefficient of involvement in sedentary leisure activity proving significant (see Table 3 ). This means that in the male sample, children who were more involved in sedentary leisure activity also had lower

Table 1. Intercorrelations among and descriptive information of body mass index (BMI), respect of rules, regularity of breakfast, active and sedentary leisure activity (males are below the diagonal)

\begin{tabular}{lccccc}
\hline & 1 & 2 & 3 & 4 & 5 \\
\hline 1. BMI & - & 0.03 & $-0.21^{* *}$ & 0.01 & $0.14^{*}$ \\
2. Respect of rules & $-0.22^{* *}$ & - & -0.07 & 0.13 & -0.03 \\
3. Regularity breakfast & -0.04 & 0.01 & - & -0.05 & -0.07 \\
4. Active Leisure & 0.06 & -0.14 & 0.04 & - & 0.01 \\
Activity & & & & & \\
5. Sedentary Leisure & 0.06 & $-0.26^{* *}$ & -0.04 & 0.05 & - \\
Activity & 16.65 & 4.86 & 3.64 & 0.77 & 0.76 \\
$M$ (males) & 2.04 & 1.04 & 0.82 & 0.42 & 0.43 \\
$S D$ (males) & 16.64 & 5.75 & 3.73 & 0.74 & 0.66 \\
$M$ (females) & 2.65 & 0.61 & 0.61 & 0.44 & 0.48 \\
$S D$ (females) & & & & & \\
\hline
\end{tabular}

Notes: $* p<0.05, * * p<0.01$

Table 2. Hierarchical regression results predicting body mass index (BMI)

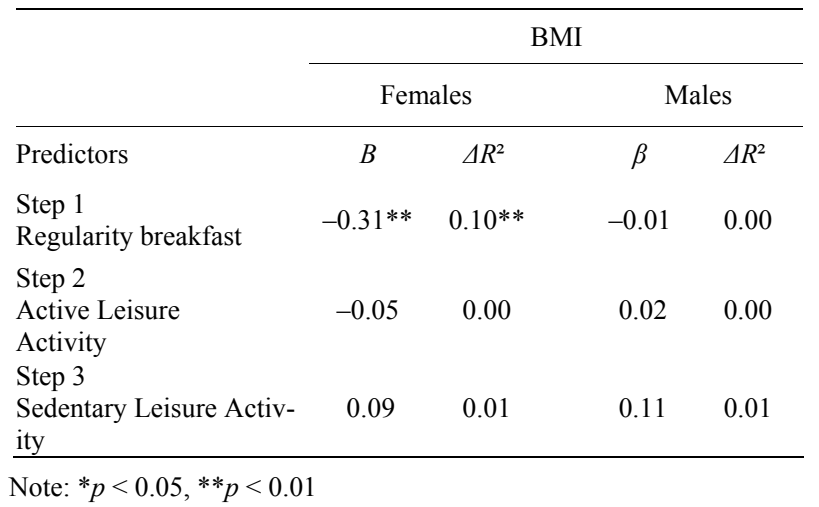

Table 3. Hierarchical regression results predicting respect of rules

\begin{tabular}{lcccc}
\hline & \multicolumn{4}{c}{ BMI } \\
\cline { 2 - 5 } & \multicolumn{2}{c}{ Females } & \multicolumn{2}{c}{ Males } \\
\hline Predictors & $B$ & $\Delta R^{2}$ & $\beta$ & $\Delta R^{2}$ \\
Step 1 & -0.01 & 0.00 & -0.09 & 0.01 \\
$\begin{array}{l}\text { Regularity breakfast } \\
\text { Step 2 }\end{array}$ & 0.11 & 0.01 & -0.14 & 0.01 \\
$\begin{array}{l}\text { Active Leisure Activity } \\
\text { Step 3 }\end{array}$ & -0.04 & 0.00 & $-0.23^{*}$ & $0.06^{*}$ \\
Sedentary Leisure Activity &
\end{tabular}

Note: ${ }^{*} p<0.05, * * p<0.01$

levels of respect of relational rules that is they were less likely to respect these rules.

No other relationship reached significance neither in the female sample, nor in the male sample.

\section{Discussion}

The present study investigated whether regularity of eating (in terms of breakfast) and spontaneous active leisure activity functioned as protective factors; that is, if they were associated to a more positive development of children in terms of both not being overweight and of being able to respect relational rules in a social context. Besides, we investigated whether sedentary spontaneous leisure activity worked as risk factor; that is if it was associated with less positive child development. Finally we explored the presence of gender differences in both the mean levels and patterns of relationships among indicators of children's adjustment and precursors of more or less healthy life-styles.

Although this was mainly an exploratory study, we expected that girls were more likely to respect relational rules and that boys were more involved in both kinds of leisure activity. Our findings confirmed the majority of these expectations: girls respected relational rules more than boys and boys were more involved in sedentary leisure activity with their friends. Nonetheless, we note that these findings must be considered with caution for the reasons we will list in the limitations section. However, we also think that these descriptive results deserve some reflection especially considering that we are unaware of previous studies on similar subjects. Firstly, we think that is interesting to observe that some gender differences that are generally found in adolescence, such as a greater tendency of boys to transgress, may seem to have quite early origins in childhood. This phenomenon probably starts within the socialization processes, which may still be likely to promote different kinds of behavior in male and female children [22]. Nevertheless, it is necessary to admit that this finding could also be related to the fact that the kinds of relational rules transgressions that the teachers of physical activity can register in their check- 
list are by definition overt behaviors. Previous research has shown that boys are generally more likely than girls to be involved in this kind of transgression behavior and that conversely girls are usually more likely than boys to be involved in covert (or relational) types of transgression of relational rules [22,23], which are difficult to register with a check-list addressed at highlighting behavioral facets of relationships.

Furthermore, we found that females and males were much more similar than expected with respect to the kind of spontaneous leisure activity they were involved in: in fact boys were more involved than girls in sedentary activities, such as playing at videogames and watching TV. This finding resembles what has been already shown for the use of the new media: boys usually will use it more often than girls [20]. We think that is again possible to interpret this finding as a result of socialization process, which could be more likely to promote the use and the experimentation of new technologies among males.

We also hypothesized to find similar relationships among the precursors of risk and protective factors regarding regularity of eating and active and sedentary leisure activities, and with the two indicators of childhood positive development (not being overweight and being able to respect relational rules) among both genders. Indeed, we found some similarities but also some differences.

Firstly the inter-correlations showed that the two indicators of adjustment were almost independent in girls; that is to say that a girl who was overweight was not more likely to also transgress relational rules. On the contrary being overweight and transgressing relational rules were strongly related in boys. Therefore our findings seems to confirm a pattern in childhood similar to what other authors have previously highlighted in adolescence between bullying and being overweight [6]. In parallel to these authors, we also think that there are alternative explanations for this phenomenon. On one hand, perceived asymmetry of power could lead more heavy male children to use their apparent strength instead of social competence in order to try to emerge from their peers [24]. On the other hand, perceived isolation from the peer group due to being overweight could stimulate the need to find alternative ways for improving one's own social acceptance in the peer group: in this case, transgressing rules could represents an attempt of becoming more interesting at the eyes of peers [25]. However, further longitudinal research is certainly needed for individuating the direction of this relationship.

Secondly, we were able to predict being overweight only in girls and being more likely to transgress relational rules only in boys. As anticipated, this may be interpreted as an indication of the differential importance of these two indicators of adjustment in boys and girls at later stages of development, such as during adolescence [18]. However, again longitudinal studies are needed for disentangling the direction of these relationships. At the present stage of this research we cannot state whether being overweight in girls is a result of irregularity of eating habits or on the contrary whether eating behavior becomes irregular as a reaction of having become previously overweight and thus an unsuccessful attempt of reducing weight. At the same extent we do not know whether transgressing relational rules in boys is the result of lack of experimentation of social competence due to abundance of sedentary behavior or on the contrary whether sedentary behavior becomes more likely a way to answer perceived discomfort in peer relationships and thus as an attempt at constructing one's own social reputation. In this latter case we would need both a longitudinal design and also different measures in order to investigate the direction of the relationships and also to evaluate the occurrence of social isolation and aggressiveness towards peers.

Thirdly, while regularity of eating was found to be related only with Body Mass Index, involvement in sedentary activity was found to be related especially with transgression of relational rules. Thus, we found almost two separate syndromes instead of the expected one, the former more concerned with the so-called internalizing problems and that seems more relevant for girls, and the latter more concerned with externalizing problems and that seems more important for boys. Although, there is some indication that internalizing problems may develop in an almost independent pattern with respect to that of externalizing problems [26], we would like to know more about their developmental paths.

\section{Strengths and Limitations}

Two key limitations of the present study are the crosssectional design and the relative weakness of some of the measures employed, although the young age of the participants forced us to use longer series of items. These two limitations precluded us from investigating the direction of the relationships we found and also to use more complex strategies of analyses which could have shed more light on the phenomenon under study. The next step in this line of research is to investigate these associations over time and also to introduce some measures of social isolation and aggressiveness. For instance, it might be useful to know whether being overweight or being more likely to transgress relational rules in childhood might contribute to the chance of bullying other people or being victimized in early adolescence for boys, and whether being overweight in childhood contributes to the further development of eating problems or other kinds of internalizing symptoms later on for girls.

The relatively small sample size and the fact that all 
participants resided in one region of Italy also makes it difficult to generalize results to different populations.

In spite of its limitations, this study has also some merits.

First, it has highlighted the importance of investigating the precursors of more or less healthy life-styles in childhood although they can assume different figures in subsequent phases of development. Second, it has underlined the importance of collocating children and not only adolescent adjustment in the complex web of relationships between personal characteristics, life contexts and behaviour. Third, it has shown that parents can do something very simple to prevent their children being overweight which is to promote regularity of their eating behaviour.

Finally, it has highlighted the potential relevance for present and future adjustment of more sedentary or more active leisure activity and not only with respect to physical condition. We are really convinced that introducing special kinds of physical activity curricular programs to children, structured in the ways suggested by Larson [9] and Fraser-Thomas, Cǒté, Deakin [16], can be really helpful to promote more positive development in children and that it can have positive consequences for their future adjustment in terms of both physical and psychological health. To implement this kind of intervention and to evaluate its effectiveness is one of our next steps.

\section{Acknowledgements}

This study was partially funded by CRC Foundation, Cuneo, Italy. We warmly thank Fulvia Gemelli, Giovanni Musella and Monica Liubicich, S.U.I.S.M., Torino, Italy, for their support and suggestions for the realisation of this research. We also thank the students of MA at the Faculty of Psychology and at S.U.I.S.M., Torino, Italy who served respectively for administering questionnaires and for evaluating the children's behaviour and Body Mass Index. Finally, we thank the children and their parents and teachers who accepted to participate at this study with great enthusiasm.

\section{REFERENCES}

[1] W. Damon, "What is Positive Youth Development?" The Annals of the American Academy of Political and Social Science, Vol. 591, No. 1, 2004, pp. 13-24.

[2] S. F. Hamilton, M. A. Hamilton and K. Pittman, "Principles for Youth Development," In: S. F. Hamilton and M. A. Hamilton, Eds., The Youth Development Handbook. Coming of Age in American Communities, Sage Publications, Inc., Thousand Oaks, 2004, pp. 3-22.

[3] C. Peterson, "Positive Social Science," The Annals of the American Academy of Political and Social Science, Vol. 591, No. 1, 2004, pp. 186-201.
[4] World Health Organization, "Global Strategy on Diet, Physical Activity and Health," WHO, Genève, 2004.

[5] G. S. Berenson, S. R. Scrinivasan, W. Bao, W. P. Newman, R. E. Tracy and W. A. Wattigney, "Association between Multiple Cardiovascolar Risk Factors and Atherosclerosis in Children and Young Adults. The Bogalusa Heart Study," The New England Journal of Medicine, Vol. 338, No. 23, 1998, pp. 1650-1656.

[6] M. Giletta, R. H. J. Scholte, R. C. M. E. Engels and J. Larsen, "Bullying Involvement among High Weight Status Adolescents," Submitted at Journal of Adolescence.

[7] R. Jessor, J. E. Donovan and F. M. Costa, "Beyond Adolescence-Problem Behavior and Young Adult Development," Cambridge University Press, Cambridge, 1991.

[8] R. Jessor, M. S. Turbin, F. M. Costa, Q. Dong, H. Zhang and C. Wang, "Adolescent Problem Behavior in China and the United States: A Cross-National Study of Psychosocial Protective Factors," Journal of Research on Adolescence, Vol. 13, No. 3, 2003, pp. 329-360.

[9] R. W. Larson, "Toward a Psychology of Positive Youth Development," American Psychologist, Vol. 55, No. 1, 2000, pp. 170-183.

[10] R. K. Silbereisen, K. Eyferth and G. Rudinger, "Development as Action in Context," Springer-Verlag, Berlin, 1986.

[11] D. Hayes and C. E. Ross, "Body and Mind: The Effect of Exercise, Overweight, and Physical Health on Psychological Well-Being," Journal of Health and Social Behavior, Vol. 27, No. 4, 1986, pp. 387-400.

[12] R. W. Larson, "Youth Organizations, Hobbies, and Sports as Developmental Contexts," In: R. K. Silbereisen and E. Todt, Eds., Adolescence in Context: The Interplay of Family, School, Peers, and Work in Adjustment, SpringerVerlag, New York, 1994, pp. 46-65.

[13] J. L. Mahoney and H. Stattin, "Leisure Activities and Adolescent Antisocial Behavior: The Role of Structure and Social Context," Journal of Adolescence, Vol. 23, No. 2, 2000, pp. 113-127.

[14] A. Bandura, "Self-Efficacy: The Exercise of Control," Freeman Publishers, New York, 1997.

[15] A. J. Huebner and J. A. Mancini, "Shaping Structured Out-of-School Time Use among Youth: The Effect of Self, Family and Friend System," Journal of Youth and Adolescence, Vol. 32, No. 6, 2003, pp. 453-463.

[16] J. L. Fraser-Thomas, J. Cŏté and J. Deakin, "Youth Sport Programs: An Avenue to Foster Positive Youth Development," Physical Education and Sport Pedagogy, Vol. 10, No. 1, 2005, pp. 19-40.

[17] B. J. Bredemeier and D. L. Shields, "Moral Growth through Physical Activity: A Structural Developmental Approach," In: D. R. Gould and M. R. Weiss, Eds., Advances in Paediatric Sport Sciences, Human Kinetics Publishers, Champaign, Vol. 2, 1987, pp. 143-165.

[18] S. Bonino, E. Cattelino and S. Ciairano, "Adolescents and Risk. Behaviors, Functions and Protective Factors," Springer 
Verlag, Milan, 2005.

[19] R. J. Brustad, "Attraction to Physical Activity in Urban Schoolchildren: Parental Socialization and Gender Influences," Research Quarterly for Exercise and Sport, Vol. 67, No. 3, 1996, pp. 316-323.

[20] J. Colwell, C. Grady and S. Rhaiti, "Computer Games, Self-Esteem, and Gratification of Needs in Adolescents," Journal of Community and Applied Social Psychology, Vol. 5, No. 3, 1995, pp. 195-206.

[21] National Institute of Statistics (ISTAT), "Annuario Statistico Italiano 2007 (Italian Statistical Yearbook 2007)," ISTAT, Rome, 2007.

[22] E. E. Maccoby and C. N. Jacklin, "Sex Differences in Aggression: A Rejoinder and Reprise," Child Development, Vol. 51, No. 4, 1980, pp. 964-980.
[23] K. Björkqvist, "Sex Differences in Physical, Verbal, and Indirect Aggression: A Review of Recent Research," Sex Role, Vol. 30, No. 3-4, 1994, pp. 1573-2762.

[24] A. D. Pellegrini, "Affiliative and Aggressive Dimensions of Dominance and Possible Functions during Early Adolescence," Aggression and Violent Behavior, Vol. 7, No. 1, 2002, pp. 21-31.

[25] S. Harter, C. Stocker and N. Robinson, "The Perceived Directionality of the Link between Approval and SelfWorth: the Liabilities of a Looking Glass. Self-Orientation among Adolescents," Journal of Research on Adolescence, Vol. 6, No. 3, 1996, pp. 285-308.

[26] K. K. Davison, M. B. Earnest and L. L. Birch, "Participation in Aesthetic Sports and Girls' Weight Concerns at Ages 5 and 7," International Journal of Eating Disorders, Vol. 31, No. 3, 2002, pp. 312-317. 